\title{
The relationship between different age swimmers' flip turn temporal and kinematic characteristics
}

\author{
Valentina SKYRIENE*, Milda DUBOSIENE**, Mindaugas DUBOSAS***, \\ Marija EIDUKEVICIUTE**** \\ *Lithuanian Sports University, Sporto 6, LT-44221 Kaunas, Lithuania, E-mail:valentina.skyriene@lsu.lt \\ **Kaunas University of Technology, Studentu 56, LT-51424 Kaunas, Lithuania, E-mail: milda.dubosiene@ktu.lt \\ ***Kaunas University of Technology, A. Mickevičiaus 37, LT-44244, Kaunas, Lithuania, \\ E-mail: mindaugas.dubosas@ktu.lt \\ ****Kaunas University of Technology, Studentu 56,LT-51424 Kaunas, Lithuania, E-mail: marija.eidukeviciute@ktu.lt
}

cross $^{\text {ref }}$ http://dx.doi.org/10.5755/j01.mech.23.4.17852

\section{Introduction}

Flip turn is very important competitive distance element at a distance swimming events. The kinematic analysis of various Olympic swimming competition events [1] suggests that the time of the turn affects the final result. The scientists who have analysed competitive swimmers' activity and the turn impact on the result [1-3] claim that by the improvement of the turn technique and shortening of its execution time, the significant improvement of competition result time is possible by $0.20 \mathrm{~s}$ per one lap. Authors [3] claim that improvement of the turn by $1 \%$ might become an important parameter separating 1 st and the 2 nd place winners in $100 \mathrm{~m}$ swimming event, thus it is important to determine the factors that might have impact on fast and effective flip turn execution.

Most of the authors have analysed the impact of the turning phase duration to the total turning time or the total competitive distance time [3-12]. These authors have determined the relationship between the turn's temporal characteristics and the impact for the total turn execution time or distance result regardless to the conditions of the flip turn execution. Pereira [9] have investigated different flip turn execution techniques and their impact on temporal characteristics, however the analysed literature did not provide any studies which assessed the impact of different conditions of flip turn execution to the temporal and kinematic characteristics of the turn.

The size of Lithuanian and foreign swimming pools differs. The only similar dimension is the length of the pool which must be of $25 \mathrm{~m}$ or $50 \mathrm{~m}$, but the depth and the width are not strictly defined. The athletes most frequently train and participate in competitions in pools of different depth (and conditions), therefore arises the question if there is the relationship between turn's temporal and kinematic characteristics considering different turn's execution conditions and different age of athletes.

The aim of the study is to determine the correlation between flip turn temporal and kinematic factors considering different turn execution conditions (in the deep and shallow side of the pool) and considering different age of the athletes.

\section{Materials and methods}

The study was performed in 2013 at the pool of Lithuanian Sports University. 24 athletes of Kaunas swimming school participated in this study. The age of the athletes varied from 12 to 22 years, their qualification varied from class I to Lithuanian Swimming Master, their height varied from 150 to $196 \mathrm{~cm}$, their body mass varied from 40 to $84 \mathrm{~kg}$. The subjects were divided into two age groups: under the age of 17 years (young swimmers) and above the age of 17 years (adult swimmers).

The swimmers' height $(\mathrm{cm})$, length of legs (from femoral head to the ground) $(\mathrm{cm})$ and body mass $(\mathrm{kg})$ were measured using anthropometry technique. The joints of hip, knee and the tarsus were marked with black water resistant marker on left and right side of the body (Fig. 1, a). Two different cases of three test flip turns were recorded: in the shallow (1.1 m) side of pool (Fig. 1, b "shallow") and deep (4.5 m) side of the pool (Fig. 1, b) "deep").

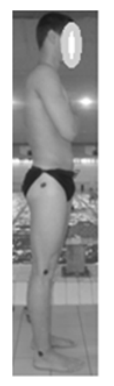

a)



b)
Fig. 1 Scheme of: a - marking of the athlete's joints; $\mathrm{b}$ - filming scheme: black lines are swimming trajectory; small black rectangles indicate flip turn position; the white rectangles on top indicate the camera location and hatched slant lines indicate camera's filming area

The underwater image was recorded using underwater video system. The system was installed permanently on the pool's edge (Fig. 1, b), underwater recording "eye" was perpendicular to the swimmer and recorded the side view from the athlete's swim in into turn until swim out of the turn. For the calibration of the underwater video system the calibration frame was filmed, consisting of vertical and horizontal bars, joined with each other by $90^{\circ}$ angle.

The recorded image from the camera is uploaded 
to the computer and cut into smaller format files using the Adobe Premiere Pro 2.0 software. The temporal and kinematic data are obtained using Simi Motion software with $50 \mathrm{~Hz}$ video player. The calculated data and its description:

1. Swim in time (s), recorded from the moment when the swimmer's head crosses the $7.5 \mathrm{~m}$ marking line before the rotation until the moment when his/her feet touch the wall.

2. Swim out time (s), recorded from the moment when feet touch the wall until the moment when the swimmer's head crosses the $7.5 \mathrm{~m}$ marking line.

3. Total turn $(7.5 \mathrm{~m}+7.5 \mathrm{~m})$ execution time $(\mathrm{s})$.

4. Rotation time (s), recorded from the moment when the head and the body are starting to bend down until the touch of the wall with the feet.

5. Contact time (s), recorded from the moment when feet touch the wall until the moment of toes withdrawal from the wall.

6. Stabilization time of the passive force (stabilization) phase - time (s), recorded from the moment of wall touch with feet until the start of legs straightening.

7. Push off time of the active force (push off) phase time (s), recorded from the start of legs straightening until the moment of toes withdrawal from the wall.

8. Gliding time of the gliding phase - time (s), recorded from the moment of the toes withdrawal from the wall until the first motion of the legs.

9. Tuck index - the ratio of the smallest distance between the hip joint and the wall (Fig. 2, a) with the total length of the leg (rel.v.). Tuck index was represented as the maximum degree of lower limb tuck during the turn. This was the distance of the hip from the wall at impact, divided by the trochanterion height [4].

10. Feet depth - the depth of the wall touch with feet (the distance of the perpendicular derived from the water surface to the tarsus bone) (m) (Fig. 2, b).

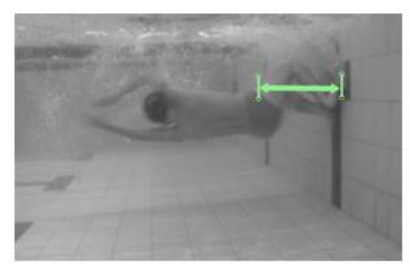

a)

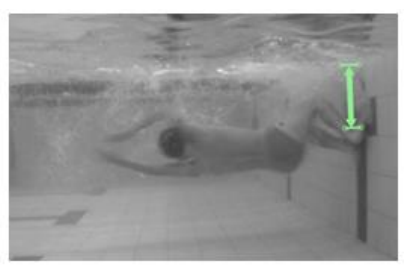

b)
Fig. 2 a - maximum leg flexion (tuck index indicator, 9); $\mathrm{b}$ - depth of wall touch with feet (feet depth indicator, 10)

Statistical analysis of the research data was made using Statistics 18 (SPSS) software. The mean values, standard deviation (SD) were calculated. The temporal and kinematic flip turn correlation was evaluated using linear Pearson correlation analysis and significant correlation at the level 0.01 and at the level 0.05 were determined.

\section{Results}

A successful swim flip turn depends on multitude of factors and requires series of complex moves to optimise the total turning performance. By this study, we aimed to determine if additional factors (pool depth) might have impact on the swimming flip turn temporary and kinematic indicators and also consider the different age of athletes. The linear correlation (Pearson) between flip turn temporal and kinematic characteristics considering different turn execution conditions and different age of the subjects (Table 1) are presented in this section. Additional statistical data of the analysed indicators: mean value, standard deviation (SD), minimum (min) and maximum (max) values are provided in Table 2.

Table 1

Correlation of swimmers turns' execution temporal and kinematic indicators when turns are executed in the deep and shallow sides of the pool

\begin{tabular}{|c|c|c|c|c|c|c|c|c|c|c|c|c|c|c|c|c|c|c|c|c|}
\hline & & & \multicolumn{9}{|c|}{ Young swimmers } & \multicolumn{9}{|c|}{ Adult swimmers } \\
\hline No. & Abbreviation & & 2 & 3 & 4 & 5 & 6 & 7 & 8 & 9 & 10 & 2 & 3 & 4 & 5 & 6 & 7 & 8 & 9 & 10 \\
\hline \multirow{2}{*}{1.} & \multirow{2}{*}{ Swim in } & $S$ & $62 * *$ & $92 * *$ & 31 &,- 16 &, 17 &, 01 &,- 42 &,- 19 & 02 & $68 *$ & $89 * *$ &, 17 &, 15 &,- 20 &,- 03 &, 34 &, $58^{*}$ &,- 10 \\
\hline & & $\mathrm{D}$ & $86 * *$ & $96 * *$ & 17 &, 35 & ,06 & 28 &,- 26 &,- 22 &,- 35 & ,54 & $86^{* * *}$ & $76^{* *}$ & 11 &,$- 82 * *$ &,- 47 & ,61* &, $68 *$ &,- 25 \\
\hline \multirow{2}{*}{2.} & \multirow{2}{*}{ Swim out } & S & & $88 * *$ & 31 &,- 08 &,- 16 &,- 13 &,- 21 & 14 &, 18 & &, $94 * *$ & \begin{tabular}{|l|}
, 12 \\
\end{tabular} & ,51 &,- 27 &, 17 & ,36 & 51 &,- 54 \\
\hline & & $\mathrm{D}$ & & $96 * *$ & 27 &, 36 &,- 02 & 24 &,- 21 &,- 10 &,- 20 & & $89 * *$ & ,27 & $65^{*}$ &,- 10 & ,38 & 13 &,- 05 &,$- 56^{*}$ \\
\hline \multirow{2}{*}{3.} & \multirow{2}{*}{ Turn } & $\mathrm{S}$ & & & 34 &,- 14 & ,02 &,- 06 &,- 36 &,- 04 &, 10 & & & \begin{tabular}{|l|}
, 14 \\
\end{tabular} & ,39 &,- 26 &, 10 &, 38 &, $59 *$ &,- 39 \\
\hline & & $\mathrm{D}$ & & & 22 &, 39 & ,03 & ,29 &,- 25 &,- 19 &,- 29 & & & ,57* & ,44 &,- 50 &,- 03 &, 41 & ,34 &,- 47 \\
\hline \multirow{2}{*}{4.} & \multirow{2}{*}{ Rotation } & $\mathrm{S}$ & & & &, 30 &,$- 53 *$ &,- 13 &,- 27 & , 48* & $65^{* * *}$ & & & &,- 45 &,- 38 &,- 53 &, 11 &, 11 &, 47 \\
\hline & & $\mathrm{D}$ & & & & $49^{*}$ &,- 18 &, 24 &,- 24 &, 21 &, $51 *$ & & & & 16 &,$- 73 * *$ &,- 37 & $90 * *$ &, $71 *$ &,- 05 \\
\hline \multirow[b]{2}{*}{5.} & \multirow{2}{*}{ Contact } & $\mathrm{S}$ & & & & & ,23 & $80 * *$ &,- 43 &,- 05 & ,54* & & & & &, 18 & $76 * *$ &,- 07 &, 09 &, $79 * *$ \\
\hline & & $\mathrm{D}$ & & & & &, 10 & $78 * *$ &,- 22 &,- 36 &, 07 & & & & &, 07 &, 76 &,- 02 & 02 &,- 61 \\
\hline \multirow{2}{*}{6.} & \multirow{2}{*}{ Stabilization } & $\mathrm{S}$ & & & & & & ,77** & ,01 &,$- 86 * *$ &,- 43 & & & & & & $78 * *$ &,- 22 &,$- 63 *$ &, 24 \\
\hline & & $\mathrm{D}$ & & & & & & $70 * *$ &,- 07 &,$- 85 * *$ &,$- 50 *$ & & & & & &, $71 *$ &,$- 62 *$ &,$- 84 * *$ &, 19 \\
\hline \multirow{2}{*}{7.} & \multirow{2}{*}{ Push off } & S & & & & & & &,- 30 &,$- 57 *$ &, 11 & & & & & & &,- 20 &,- 35 &,- 34 \\
\hline & & $\mathrm{D}$ & & & & & & &,- 20 &,$- 80 * *$ &,- 27 & & & & & & &,- 41 &,- 52 &,- 33 \\
\hline \multirow{2}{*}{8.} & \multirow{2}{*}{ Gliding } & $\mathrm{S}$ & & & & & & & &,- 09 &,- 40 & & & & & & & &, 42 &,- 12 \\
\hline & & $\mathrm{D}$ & & & & & & & &, 13 &, 08 & & & & & & & &, 50 &,- 11 \\
\hline \multirow{2}{*}{9} & \multirow{2}{*}{ Tuck index } & $S$ & & & & & & & & &, $53 *$ & & & & & & & & &,- 47 \\
\hline & & $\mathrm{D}$ & & & & & & & & & ,54* & & & & & & & & &,- 05 \\
\hline 10. & Foot depth & & & & & & & & & & & & & & & & & & & \\
\hline
\end{tabular}

Note: **. Correlation is significant at the 0.01 level (1-tailed).

*. Correlation is significant at the 0.05 level (1-tailed).

$\mathrm{S}$ - Shallow and D - Deep side of the pool. 
Additional statistical data of the analysed indicators: mean value, standard deviation (SD), minimum (min) and maximum (max) values

\begin{tabular}{|c|c|c|c|c|c|c|c|c|c|c|}
\hline \multirow{2}{*}{ No. } & \multirow{2}{*}{ Abbreviation } & & \multicolumn{4}{|c|}{ Young swimmers } & \multicolumn{4}{|c|}{ Adult swimmers } \\
\hline & & & $\begin{array}{c}\min . \\
(\mathrm{s})\end{array}$ & $\begin{array}{c}\max . \\
(\mathrm{s})\end{array}$ & $\begin{array}{c}\text { mean } \\
\text { (s) }\end{array}$ & $\begin{array}{l}\text { SD } \\
\text { (s) }\end{array}$ & $\begin{array}{c}\min . \\
(\mathrm{s})\end{array}$ & $\begin{array}{c}\max . \\
\text { (s) }\end{array}$ & $\begin{array}{c}\text { mean } \\
\text { (s) }\end{array}$ & $\begin{array}{l}\text { SD } \\
\text { (s) }\end{array}$ \\
\hline \multirow{2}{*}{1.} & \multirow{2}{*}{ Swim in } & $\mathrm{S}$ & 4,62 & 5,96 & 5,44 & 0,32 & 4,29 & 5,0 & 4,72 & 0,25 \\
\hline & & $\mathrm{D}$ & 4,79 & 6,38 & 5,51 & 0,36 & 4,36 & 5,22 & 4,79 & 0,27 \\
\hline \multirow{2}{*}{2.} & \multirow{2}{*}{ Swim out } & $\mathrm{S}$ & 3,54 & 4,39 & 4,02 & 0,28 & 3,11 & 4,39 & 3,58 & 0,34 \\
\hline & & $\mathrm{D}$ & 3,35 & 4,69 & 4,11 & 0,37 & 3,09 & 4,19 & 3,55 & 0,29 \\
\hline \multirow{2}{*}{3.} & \multirow{2}{*}{ Turn } & $\mathrm{S}$ & 8,15 & 10,35 & 9,45 & 0,54 & 7,62 & 9,4 & 8,3 & 0,54 \\
\hline & & D & 8,14 & 11,06 & 9,6 & 0,7 & 7,83 & 9,42 & 8,34 & 0,49 \\
\hline \multirow{2}{*}{4.} & \multirow{2}{*}{ Rotation } & $\mathrm{S}$ & 0,84 & 1,23 & 0,99 & 0,1 & 0,85 & 1,21 & 0,97 & 0,1 \\
\hline & & $\mathrm{D}$ & 0,76 & 1,33 & 1,05 & 0,16 & 0,87 & 1,25 & 1,05 & 0,14 \\
\hline \multirow{2}{*}{5.} & \multirow{2}{*}{ Contact } & $\mathrm{S}$ & 0,25 & 0,47 & 0,33 & 0,07 & 0,18 & 0,35 & 0,29 & 0,06 \\
\hline & & $\mathrm{D}$ & 0,23 & 0,43 & 0,3 & 0,07 & 0,16 & 0,34 & 0,28 & 0,05 \\
\hline \multirow{2}{*}{6.} & \multirow{2}{*}{ Stabilization } & $\mathrm{S}$ & 0,05 & 0,2 & 0,11 & 0,05 & 0,04 & 0,15 & 0,09 & 0,04 \\
\hline & & $\mathrm{D}$ & 0,05 & 0,26 & 0,1 & 0,05 & 0,03 & 0,13 & 0,08 & 0,03 \\
\hline \multirow{2}{*}{7.} & \multirow{2}{*}{ Push off } & $\mathrm{S}$ & 0,15 & 0,31 & 0,22 & 0,04 & 0,14 & 0,25 & 0,2 & 0,04 \\
\hline & & $\mathrm{D}$ & 0,14 & 0,31 & 0,19 & 0,05 & 0,13 & 0,25 & 0,19 & 0,03 \\
\hline \multirow[b]{2}{*}{8.} & \multirow{2}{*}{ Gliding } & $S$ & 0,09 & 0,4 & 0,26 & 0,08 & 0,06 & 0,68 & 0,24 & 0,19 \\
\hline & & $\mathrm{D}$ & 0,04 & 0,41 & 0,24 & 0,09 & 0,05 & 0,48 & 0,21 & 0,16 \\
\hline \multirow{2}{*}{9.} & \multirow{2}{*}{ Tuck index } & $\mathrm{S}$ & 0,49 & 0,85 & 0,68 & 0,1 & 0,59 & 0,86 & 0,71 & 0,09 \\
\hline & & $\mathrm{D}$ & 0,52 & 0,78 & 0,67 & 0,09 & 0,55 & 0,85 & 0,71 & 0,09 \\
\hline \multirow{2}{*}{10.} & \multirow{2}{*}{ Foot depth } & $S$ & 0,26 & 0,56 & 0,34 & 0,08 & 0,18 & 0,42 & 0,34 & 0,07 \\
\hline & & $\mathrm{D}$ & 0,19 & 0,53 & 0,37 & 0,09 & 0,2 & 0,4 & 0,35 & 0,07 \\
\hline
\end{tabular}

Note: $\mathrm{S}$ - shallow side of swimming pool; $\mathrm{D}$ - deep side of swimming pool.

Despite different conditions for the flip turn execution (in deep and shallow sides of the pool) and different age of the subjects, average and strong statistically significant positive correlation $(p<0.01, p<0.05)$ was detected between the $7.5 \mathrm{~m}$ swim in time and $7.5 \mathrm{~m}$ swim out time and also with total turn execution time (except for adult swimmers swim in and swim out times in deep side of the poolthey did not correlate). Also despite different age of the athletes and pool depth, the times of the contact and stabilization phases had strong positive correlation with push-off phase time, though stabilization phase time had negative correlation with tuck index.

The kinematic parameters of flip turn executed by young swimmers correlated with temporal characteristics of turn phases. According the turn data at the deep pool side, the positive correlation between feet depth and rotation phase time and also with tuck index was determined. At the same conditions the negative correlation was determined between the feet depth and stabilization phase time. At the shallow side of the pool, the feet depth data positively correlated with the times of rotation phase and contact phase, and also with tuck index. The negative correlation between tuck index and the times of stabilization phase and push-off phase was determined for both pool sides. Tuck index positively correlated with rotation phase time for shallow pool side data. Whereas the positive correlation between the time of rotation phase and the time of contact phase was determined for the deep pool side.

Negative moderate correlation was determined between the time of rotation phase and stabilization phase at the shallow side of the pool and positive moderate correlation was determined between the times of rotation phase and contact phase at the deep side of the pool.

In case of the adult swimmers the flip turn temporal characteristics correlated with (kinematic) feet depth and tuck index parameters. The negative correlation was determined between feet depth and swim out time for the shallow pool side data. Whereas the positive correlation was determined between the tuck index and swim in time for the data of both sides of pool. The positive correlation was also determined between tuck index and total flip turn time at the shallow pool side and between tuck index and rotation time at the deep side of the pool.

The correlation between the temporal characteristics of flip turn phases was determined only for the deep pool side data. The rotation time had moderate correlation with the times of swim in and total turn. The wall contact time also had moderate correlation with the swim out time. The negative correlation was determined between stabilization phase time and the times of swim in and rotation phase. The gliding phase time positively correlated with swim in time and rotation phase time. The negative correlation was determined between the same gliding phase time and stabilization phase time.

\section{Discussions}

This study aimed to find out if the pool depth may influence the correlation between temporal and kinematic characteristics of different age swimmers. In order to simplify the analysis the data was grouped: the first three (swim in, swim out and turn) characteristics are referred as temporal characteristics of the flip turn, other five (rotation, contact, stabilization, push off and gliding) are referred as temporal characteristics of flip turn phases and the last ones (tuck index and feet depth) are referred as kinematic characteristics of flip turn.

Temporal characteristics of the flip turn. The flip turn analysis at the 2000 Olympic Games showed that the fastest free style swimmers were not necessarily the fastest turners [1]. Other authors claim that the improvement of the 
flip turn just by $1 \%$ might be significant parameter separating the swimmers of the 1 st and the 2 nd place [3]. The total flip turn $(15 \mathrm{~m})$ time recorded in Araujo [13] was $8.98 \pm$ $1.12 \mathrm{~s}$. The total flip turn time of this study is slightly longer for young athletes (in the shallow side of the pool it is 9.45 $\pm 0.54 \mathrm{~s}$ and in the deep side of the pool the same time is 9.6 $\pm 0.7 \mathrm{~s}$ ) and slightly shorter for adult swimmers (in the shallow side the total time is $8.3 \pm 0.54 \mathrm{~s}$ and in the deep side the total time is $8.34 \pm 0.49 \mathrm{~s}$ ).

The analysis of correlation between the temporal characteristics of the flip turn shows that the total flips turn execution time relates to the times of swim in and swim out. Regarding the aim of the study it was determined that the depth of the pool had no impact on the correlation of flip turn's temporal characteristics (except for the swim in and swim out times of the adult swimmers in the deep side of the pool as they did not correlate).

Temporal characteristics of the flip turn phases. In this study the time of the contact phase was distinguished into acting times of the passive (stabilization) and active (push off) forces. The authors who have completed the biomechanical analysis have recorded different phase times: $0.23 \pm 0.04 \mathrm{~s}[11] ; 0.32 \pm 0.04 \mathrm{~s}$ [14]; $0.41 \pm 0.11 \mathrm{~s}$ [13]. The contact phase times of this study best correspond to the results of [14]: the recorded results of the young swimmers are $0.33 \pm 0.07 \mathrm{~s}$ (deep) and $0.3 \pm 0.07 \mathrm{~s}$ (shallow), the results of the adult swimmers $0.28 \pm 0.05 \mathrm{~s}$ (deep) $0.29 \pm 0.06 \mathrm{~s}$ (shallow).

The results of the study revealed that despite the depth of the pool and different age of the athletes the shorter time of the contact with the wall relates to the shorter phase of the push-off from the wall. Whereas the time of push-off depends on stabilization time, thus both components of this phase (i.e. stabilization and push-off) have impact on the contact phase time. According authors [15], in order to optimise the wall contact time, it is important to decrease the acting time of the passive force (stabilization) and increase the acting time of the active force (push-off) by maintaining the total wall contact time as short as possible. The study data has showed that stabilization time (for young athletes in the shallow side of the pool; for the adult athletes in the deep side of the pool) relates to the rotation time, i.e. the longer is the rotation phase, the shorter is the legs stabilization and thus because of the latter the push-off time is shorter too.

Wall push-off time mostly determine swimmers speed straightaway after the push-off from the wall, i.e. in the gliding phase [15]. The author claims that the longer push-off from the wall leads to greater swimmer speed after the push-off (i.e. in the gliding phase).

According to the data of [6] the push-off time is up to $0.22 \pm 0.05 \mathrm{~s}$. The results of this study are very similar: the push off times for the young swimmers are $0.22 \pm 0.04$ $\mathrm{s}$ (shallow) and $0.19 \pm 0.05 \mathrm{~s}$ (deep), and for the adult swimmers: $0.2 \pm 0.04 \mathrm{~s}$ (shallow) and $0.19 \pm 0.03 \mathrm{~s}$ (deep). Therefore the push off time cannot last too long and, according to [5], the optimal ratio between the push-off time and the acquired speed shall be found. The highest push-off velocity will be achieved when the swimmer will execute the perfect combination: low peak drag force, high peak propulsive force and a wall push-off time of sufficient period to develop this force are required [6]. Though the author claims that the wall push-off time best determines the swimmers speed in gliding phase, the results of this study shows that stabilization time positively correlates with push-off phase time; the push-off phase time also positively correlates with the total time of the contact phase. This suggests that the whole contact phase performance influence the swimmer's velocity after the push-off and does not depend on the depth of the pool.

The results of this study also showed that the gliding phase time of the adult swimmers depends (positively correlates) on swim in time and (negatively correlates) on stabilization phase time only in the deep side of the pool, whereas in the shallow side of the pool and for the flip turns of the young swimmers the correlation between gliding phase time and other parameters was not detected.

The depth of the pool influenced some temporal characteristics of the turn phase and the total turn characteristics of the adult swimmers. The correlation was determined only between the total turn execution time (positive correlation) and rotation phase time, between the rotation phase time and swim in time. Whereas the swim in time negatively correlated with stabilization phase time. The swim out time was influenced by the time of the wall contact phase. The determination of the correlation between the temporal turn characteristics only in the deep side of the pool and only for the adult swimmers shows that the turn phase times of the athletes are influenced by pool depth and athletes' age.

Kinematic characteristics of the turn. During the turn execution the feet depth and tuck index are important kinematic parameters determining the quality of the turn execution. Thus it is important to analyse the influence of these variables to the characteristics of the turn and turn phases.

The different pool depth and athletes age influenced the correlation between tuck index and rotation phase time. Statistically significant positive correlation between the mentioned parameters for young swimmers was determined for the data from the shallow side of the pool and for the adult swimmers it was determined for the data in the deep side of the pool. It suggests that in case of the longer rotation phase, the wall is touched by the less tucked legs. This can be explained that when making the turn with straighter legs, the larger tuck index is achieved but it takes longer to throw legs in rotation phase time.

The study results showed that independently from the pool depth and athletes age, tuck index negatively correlates with stabilization phase time. It should be mentioned that if the legs are put at the greater distance from the wall considering the tuck of the legs, then the stabilization phase is shorter. For the young swimmers despite the depth of the pool, the negative correlation between the tuck index and push-off phase time was determined. That means that straighter legs shorten the wall push-off time. These results reflects the claim of the Blanksby [4] that straighter legs (with bigger distance from the wall to swimmer's femoral head), contacting the wall with them influenced the shorter push-off time and vice versa - more tucked legs have influenced the longer push-off time and also prolonged the execution of flip turn $[4,16]$. But according other authors, it is very important to evaluate the work of the muscles during the legs tuck and straightening as too much tucked or too much straightened legs can negatively influence the total result of the flip turn [17]. The authors suggest the optimal value of the tuck index of 0.46 to develop optimal push-off velocity. However the value of tuck index calculated during 
the study by Prins [17] was $0.57 \pm 0.14$. The bigger values of tuck index determined in this study (for adult swimmers independent from the depth of the pool $0.71 \pm 0.09$ and for young swimmers in the shallow side of the pool $0.68 \pm 0.1$, and in the deep side of the pool $-0.67 \pm 0.09$ ) demonstrates that the athletes performed the flip turn with straighter legs than it is recommended by the mentioned authors and the tuck index bigger than 0.46 had not ensure the optimal pushoff velocity. But this data is significant only for young athletes as in this case the ratio was statistically significant. For adult swimmers the correlation between tuck index and push-off phase time was not statistically significant.

The statistically significant positive correlation between the tuck index and swim in time was determined among adult swimmers. As the swim in time influences the rotation phase time, and the latter influences tuck index, thus swim in time also influences tuck index. As statistically significant correlation was recorded both in the deep and in the shallow side of the pool, this demonstrates that the pool depth did not influence the correlation between the tuck index and swim in time.

The results show that the pool depth influenced the correlation between feet depth and swim out time only among the adult swimmers. Maglischo [2] recommends that feet hit the wall at a depth of approximately 30 to 40 centimetres to ensure a horizontal push-off. The results of the athletes who participated in this study meet the recommendations of the author (in the shallow side of the pool for young swimmers the result was $0.34 \pm 0.08 \mathrm{~m}$, for the adult swimmers the result was $0.34 \pm 0.07 \mathrm{~m}$. In the deep side of the pool the result of young swimmers was $0.37 \pm 0.09 \mathrm{~m}$, and in the deep side of the pool $-0.35 \pm 0.07 \mathrm{~m}$.). But the results also showed that the feet depth differed in the deep and shallow sides of the pool: the feet depth for young athletes was $0.03 \mathrm{~m}$ deeper, and for the adult swimmers it was $0.01 \mathrm{~m}$ deeper. Such bigger difference between the feet depth in the deep and the shallow sides of the pool among the young athletes may be the result of the lesser experience in making the turns. The cause may also be the different depth of the pool used during the training than used for the study.

The difference between the pool depth did not influence the correlation between the feet depth and contact phase time for adult swimmers. It was determined that the deeper is feet depth, the shorter is the wall contact phase time. Though for the young swimmers the reverse case was determined in the shallow side of the pool. The deeper was the feet depth, the longer was contact phase; and in case of deeper feet depth the legs also were more tucked in both cases of the pool depth. The cause of these results might be that the swimmers swim too near to the wall and thus the legs are more tucked and the push-off performance is prolonged together with the wall contact time. The mentioned ratio was not determined in the deep side of the pool, thus it suggests that young athletes due to lack of experience compared to adults were not able properly assess the changed conditions of the pool and kinematic parameters depends on the depth of the pool where they were training.

\section{Conclusions}

The different conditions of the pool have different impact on the correlation between temporal and kinematic characteristics of different age swimmers' turn and turn phases. In case of young athletes the bigger pool depth influence was determined for kinematic turn parameters, and in case of adult athletes, the bigger pool depth influence was determined for temporal characteristics of the turn.

\section{References}

1. Mason B.R.; Cossor J.M. 2001. Swimming Turn Performance at the Sydney 2000 Olympic Games. Biomechanics Symposia 2001. University of San Francisco.

2. Maglischo E. 2003. Swimming Fastest. The essential reference on technique, training and program design. Champaign, Illinois: Human Kinetics Publishers.

3. Slawson S.; Conway P.; Justham L.; Sage T.Le.; West A. 2010. Dynamic signature for tumble turn performance in swimming. 8th Conference of the International Sports Engineering Association (ISEA), 33913396. http://dx.doi.org/10.1016/j.proeng.2010.04.163.

4. Blanksby B.A.; Gathercole D.G.; Marshall R.N. 1996. Force plate and video analysis of the tumble turn by age-group swimmers. Journal of Swimming Research. 11: 40-45.

5. Blanksby B.A.; Skender S.; Elliott B.; McElroy K. Landers G. 2004. An analysis of the rollover backstroke turn by age-group swimmers. Sports Biomechanics.3(1):1-14.

http://dx.doi.org/10.1080/14763140408522826.

6. Lyttle A.D.; Blanksby B.A.; Elliott B.C.; Lloyd D.G. 1999. Investigating kinetics in the freestyle flip turn push-off. Journal of Applied Biomechanics. 15(3): 242252.

http://dx.doi.org/10.1123/jab.15.3.242.

7. Pereira S.M.; Vilar S.; Gonçalves P.; Fernandes S.; Fernandes R.; Roesler H.; Vilas-Boas. J.P. 2007. Electromyographic analysis of the flip turn technique. XXV ISBS Symposium 2007, Ouro Preto - Brazil, $55-58$.

8. Pereira S.; Vilar S.; Gonçalves P.; Figueiredo P.; Fernandes R.; Roesler H.; Vilas-Boas J.P. 2008. A combined biomechanical analysis of the flip turn technique. ISBS Conference 2008: Coaching and Sports Performance, Seoul, Korea, 699-702.

9. Pereira S. M.; Ruschel C.; Souza T.G.; Araujo L.G.; Gonçalves P.; Fernandes R.; Roesler H.; Vilas-Boas J.P. 2011. Comparative analysis of temporal parameters of different techniques of the freestyle flip turn. Portuguese Journal of Sport Sciences, 11 (Suppl. 2): 359-362.

10. Shahbazi M.M.; Sanders R.H.; Tonddast N.S. 2006. A mathematical simulation to study pike turn characteristics in front crawl swim. XXIV ISBS Symposium 2006, Salzburg - Austria, 1-4.

11. Shahbazi M.M.; Sanders R.H.; McCabe C.; Adams D. 2007. Investigating correliations between swim pike turn kinematics variables in front crawl. XXV ISBS Symposium 2007, Ouro Preto - Brazil, 381-384.

12. Shahbazi M.M.; Sanders R.H. 2008. A new mathematical simulation to study flip turn characteristics in front 
crawl swim. ISBS Conference 2008: Coaching and Sports Performance, Seoul, Korea, 156-159.

13. Araujo L.; Pereira S.; Gatti R.; Freitas E.; Jacomel G.; Roesler H.; Villas-Boas J. 2010. Analysis of the lateral push-off in the freestyle flip turn. Journal of Sports Sciences, 28(11): 1175-1181. http://dx.doi.org/10.1080/02640414.2010.485207.

14. Lyttle A.D.; Blanksby B.A.; Elliott B.C.; Lloyd D.G. 1998. The effect of depth and velocity on drag during the streamlined glide. Journal of Swimming Research. 13:15-22.

15. Blanksby B. 1999. Gaining on Turns. XVII International Symposium on Biomechanics in Sports, 11-20. Perth, WA: School of Biomedical and Sports Science, Edith Cowan University.

16. Takahashi G.; Yoshida A.; Tsubakimoto S.; Miyashita M. 1982. Propulsive Forces Generated by Swimmers during a Turning Motion. In A. P. Hollander et al. (Eds.), Biomechanics and Medicine in Swimming: Proceedings of the Fourth International Symposium of Biomechanics in Swimming. Champaign, Ill.: Human Kinetic Publishers, 192-198.

17. Prins J.; Patz A. 2006. The Influence of Tuck Index, Depth of Foot-Plant, and Wall Contact Time on the Velocity of Push-Off in the Freestyle Flip Turn. Xth International Symposium on Biomechanics and Medicine in Swimming, 82-85.
V. Skyriene, M. Dubosiene, M. Dubosas, M. Eidukeviciute

\section{THE RELATIONSHIP BETWEEN DIFFERENT AGE SWIMMERS' FLIP TURN TEMPORAL AND KINEMATIC CHARACTERISTICS}

S u m m a r y

This paper analyses the data gathered by measuring the parameters of flip turn executed by 24 swimmers of Kaunas Swimming School. The turning tests were filmed underwater in the deep and shallow side of the pool. The main purpose was to determine relationship between the temporal and kinematic indicators. The correlation parameters varied considering different turn execution conditions and the age of the athlete. The significant correlation was determined between $7.5 \mathrm{~m}$ swim in time and $7.5 \mathrm{~m}$ swim out from the turn time. It was determined that the longer young athletes took in rotational phase, the longer was the leg stabilization in a deep side of the pool. In the shallow side of the pool the shorter time of swim in of the turn adults took shorter time in turn, push off and gliding phases.

Keywords: swimming, flip turn, kinematic characteristics, depth of the pool. 